\title{
Spin Label Study of the Factors Affecting Molecular Motion of Poly(methyl methacrylate)
}

\author{
Shigetaka Shimada, ${ }^{\dagger}$ and Keiichi Kashima \\ Nagoya Institute of Technology, Gokiso-cho, Showa-ku, Nagoya 466, Japan
}

(Received January 18, 1996)

\begin{abstract}
The electron spin resonance (ESR) line shape of nitroxide radical labels attached to poly(methyl methacrylate) (PMMA) chains was studied as a function of temperature. In order to clarify various factors affecting molecular motions of PMMA chains, molecular mobility of a spin-labeled PMMA chain (L-chain) in another PMMA (M-chain) matrix was estimated by the ESR method. Isotactic and atactic PMMA having narrow molecular weight distribution were used as L- and M-chains. Strong dependencies of molecular mobility on molecular weights $\left(M_{\mathrm{L}}\right.$ and $\left.M_{\mathrm{M}}\right)$ of the chains and tacticity of $\mathrm{M}$-chains were found. For example, the transition temperature, $T_{50 \mathrm{G}}$ at which the extrema separation width due to anisotropic hyperfine splitting drops sharply increases with increasing molecular weight of M-chains. The correlation times at the transition temperatures, calculated from the ESR spectra are compared with those in so-called relaxation map and the nitroxide rotational motion is concluded to strongly correlate with micro-Brownian motion of the PMMA chains. It was also found that the correlation times estimated by the ESR method is proportional to $M_{\mathrm{L}}^{0.5}$ and $M_{\mathrm{M}}$ when $M_{\mathrm{M}}<2 M_{\mathrm{e}}$ and independent of $M_{\mathrm{M}}$ when $M_{\mathrm{M}}>2 M_{\mathrm{e}}$. Here, $M_{\mathrm{e}}$ is the molecular $M_{\mathrm{e}}$ weight between entanglements. These experimental facts are reflected on Stokes-Einstein and reptation types of diffusions of L-chains.

KEY WORDS Electron Spin Resonance/Spin Label/Molecular Motion / Poly(methyl metacrylate) Relaxation Map / Distribution of Correlation Time /
\end{abstract}

It has been well established that spin-label technique is very useful to characterize molecular motion in solid polymers. ESR method is a simple and effective way of determining molecular mobility on a time scale of $\sim 10^{-9} \mathrm{~s}$. We developed spin-label technique to investigate the structure and dynamics behavior of polymer chain at a particular site in a complicate system such as polymer blends. ${ }^{1}$

Many authors have studied molecular motion of poly(methyl metacrylate) (PMMA) by the spin-label method. Veksli et al., ${ }^{2}$ Bullock et al., ${ }^{3}$ Shiotani et al., ${ }^{4}$ and Tsay et al. ${ }^{5}$ have analyzed the observed spectra based on the assumption that the rotational motion relies upon the simplified expression of correlation time, $\tau_{\mathrm{R}}$ proposed by Freed et al. $^{6}$ for isotropic rotation in the slowmotional region and Kivelson ${ }^{7}$ for isotropic rotation in the motional narrowing region.

On the other hand, Shiotani et al. ${ }^{8}$ concluded the anisotropic rotation of the side chain from the detailed analysis of the ESR spectrum of the spin-labeled PMMA. Murakami et al. ${ }^{9}$ and Tsay et al. ${ }^{10}$ presented spectral evidence to indicate that the double-peak feature of ESR spectrum observed for the spin-labeled PMMA can be explained satisfactorily on the basis of free-volume present in the glassy state. Cameron et al. ${ }^{11}$ also interpreted the double-peak feature of the ESR spectrum of a nitroxide spin probe as reflecting from a distribution of correlation time arising from a distribution of freevolume within a polymer matrix. On the other hand, the correlation time of the molecular motion contributing to the averaging of the magnetic parameters have been ambiguously estimated from the ESR and NMR spectra observed for solid polymers. The most convenient methods for estimating the correlation time are to apply Freed, Kivelson and so-called BPP (Bloembergenm Purcell, and Pound) equations. ${ }^{12}$ These equations are

\footnotetext{
† To whom correspondence should be addressed.
}

valid for random motion with a single correlation time. Therefore, the activation energy obtained from the temperature dependence of correlation time by use of the three equations is very small compared with the energy obtained with consideration of the distribution of correlation time in solid polymers.

We estimated the distribution from simultaneous observation of ESR and NMR spectra and concluded that the correlation time calculated from the ESR and NMR line shapes at transition temperatures, for example, $T_{50 \mathrm{G}}$, is absolutely true. ${ }^{13,14}$

In the present paper, we first clarify various factors affecting molecular motion of PMMA chains and next relate the distribution of the chemical structure with distributions of free-volume and correlation time from the temperature dependence of ESR spectra of spinlabeled PMMA in the various matrices. Finally, we present a mechanism of molecular motion of PMMA detected by the spin-label method.

\section{EXPERIMENTAL}

\section{Materials}

Many kinds of isotactic PMMA were prepared by anionic polymerization of MMA monomer (Tokyo Kasei Co., Ltd.) using tert-butyl magnesium bromide catalyst at $-78^{\circ} \mathrm{C}$. ${ }^{15}$ Three kinds of spin-labeled and isotactic PMMA were also prepared by anionic copolymerization of the MMA monomer with a labeled monomer, ${ }^{16}$ 4-(methacryloyloxy)-2,2,6,6-tetramethylpiperidine using tert-butyl magnesium bromide catalyst at $-78^{\circ} \mathrm{C}$. The end part of the PMMA chain is spin-labeled by injection of label monomer after polymerization of MMA monomer. The labeled polymer is named as the endlabeled PMMA. The mid-point of the PMMA chain is also spin-labeled by injection of MMA monomer after the preparation of the end-label PMMA. The labeled polymer is named as the center-label PMMA. On the 
Table I. Names and molecular weights of PMMA used in the present study

\begin{tabular}{clcc}
\hline & Name & $M_{n}$ & $M_{w} / M_{n}$ \\
\hline $\begin{array}{c}\text { Isotactic PMMA } \\
\text { End label }\end{array}$ & LE & $5.0 \times 10^{3}$ & 1.21 \\
Center label & LC & $5.7 \times 10^{3}$ & 1.27 \\
& HC & $6.8 \times 10^{4}$ & 1.26 \\
Random label & HR & $3.6 \times 10^{4}$ & 1.32 \\
& HRB & $3.0 \times 10^{4}$ & 4.20 \\
Non label & LN & $4.6 \times 10^{3}$ & 1.18 \\
& HN & $6.9 \times 10^{4}$ & 1.28 \\
\hline \multirow{2}{*}{ Atactic PMMA } & & & \\
& C03 & $3.0 \times 10^{3}$ & 1.07 \\
& C1 & $1.1 \times 10^{4}$ & 1.11 \\
Non label & C5 & $5.3 \times 10^{4}$ & 1.07 \\
& C60 & $5.9 \times 10^{5}$ & 1.04 \\
& C130 & $1.3 \times 10^{6}$ & 1.06 \\
& C4B & $4.0 \times 10^{4}$ & 1.85 \\
\hline
\end{tabular}

other hand, polymerization is begun in the coexistence of MMA and label monomer in order to obtain the sample named as the random-label PMMA, which is spin-labeled at random in the chain. The toluene solution of the PMMA sample is dropped into cyclohexane in order to remove the non-reactive MMA and label monomers. The sediment is dried under vacuum for more than 1 day at room temperature. The procedure is repeated three times. The reaction time and the content of MMA monomer are changed to obtain the sample with different molecular weight. Many kinds of atactic polymer were purchased from Aldrich Chemical Co., Ltd. and Polymer Sci. Product Inc. Table I shows the characterization and name code of the PMMA samples.

\section{Preparation of Blends}

Binary blend of spin-labeled and non spin-labeled PMMA were made by mixing in a ratio of $1: 20$ by weight. Films were prepared by casting $\sim 3 \mathrm{wt} \%$ of benzene solution at $60^{\circ} \mathrm{C}$ and then dried under vacuum for more than 1 week at the same temperature.

\section{ESR Measurements}

Samples were stacked in ESR sample tubes evacuated to $10^{-5} \mathrm{mmHg}$. ESR measurements were carried out with a JEOL FE\#XG spectrometer with a connected MELCOM 70/25 computer.

The signal of diphenylpicryl-hydrazyl (DPPH) was used as a $g$-value standard. The magnetic field sweep was calibrated with known splitting constants of $\mathrm{Mn}^{2+}$.

\section{GPC Measurements}

Molecular weight and its polydispersity were characterized by gel permeation chromatography (GPC). The GPC measurements were carried out with a GMXG1000-G2000-G4000 type apparatus (Tosoh Co., Ltd.).

\section{RESULTS AND DISCUSSION}

Factors Affecting Molecular Motions of PMMA Chains Figure 1 shows an example of temperature dependence of ESR spectrum of spin-labeled PMMA. Although the ESR spectrum at low temperatures was a broad triplet,

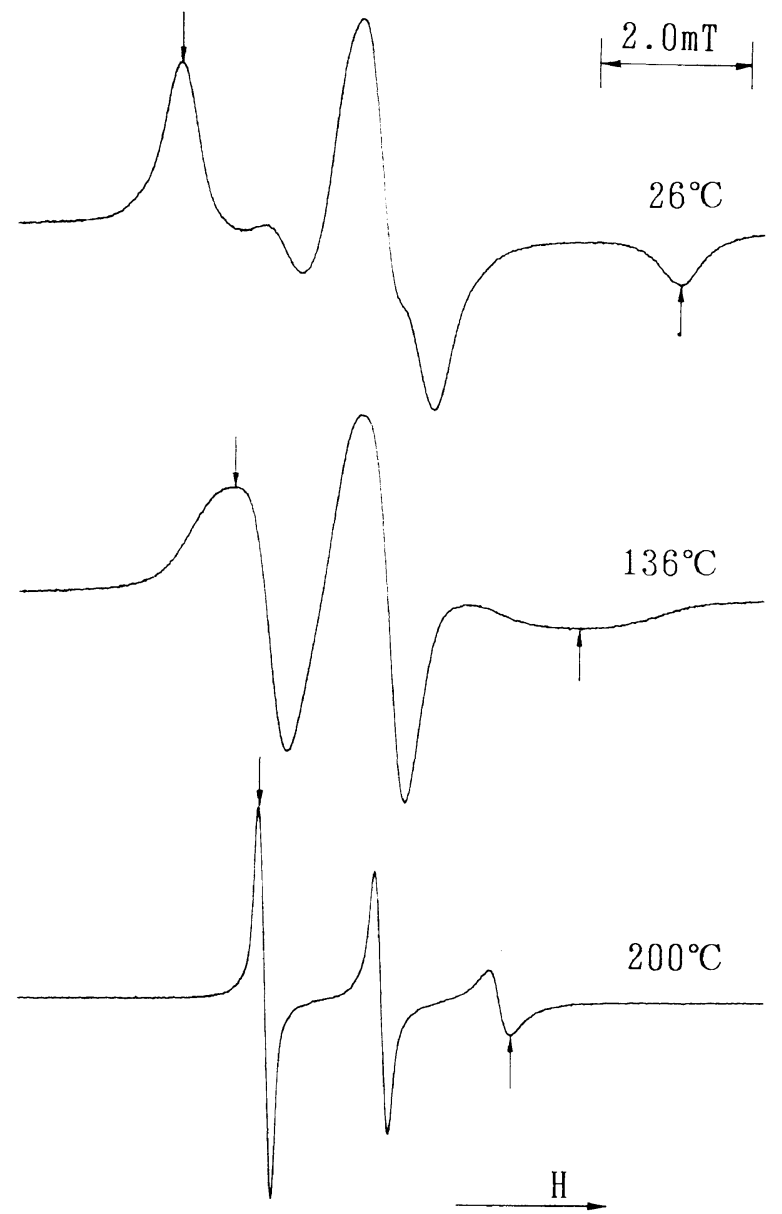

Figure 1. ESR spectra of spin-labeled PMMA (LE) in the PMMA matrix of HN. The arrows indicate the outermost peaks.

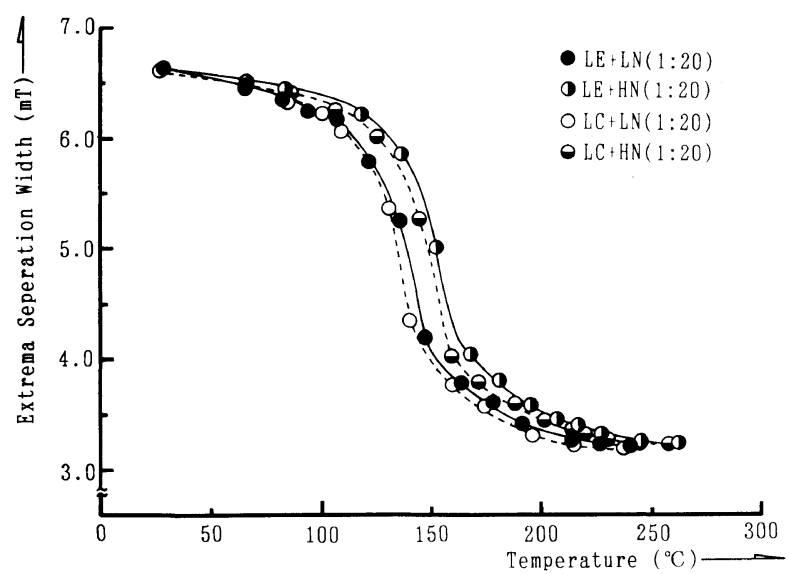

Figure 2. Variation of extrema separation width $\left(2 A_{Z}\right)$ with temperature: End-label (LE)/non-label (LN) (O); end-label (LE)/nonlabel $(\mathrm{HN})(\mathrm{O})$; center-label $(\mathrm{LC}) /$ non-label $(\mathrm{LN})(\mathrm{O})$; center-label $(\mathrm{LC}) /$ non-label $(\mathrm{HN})(\ominus)$.

a narrow component at higher temperatures appears. The width between the outermost peaks of the spectrum narrowed with increasing temperature because of averaging of the anisotropic hyperfine coupling due to rotational motion of the PMMA chains. The outermost splitting is a good measure of the mobility of the spinlabeled PMMA. Figure 2 shows examples of variation of extrema separation width with temperature. The outermost peaks gradually change in the lower temperature range and narrowed sharply at a characteristic 
temperature. In the present paper, we call the inflection point as $T_{50 \mathrm{G}}$, at which the outermost splitting is 50 gauss. The estimated temperature and the correlation time at the temperature should be closely related to glass transition temperature $\left(T_{\mathrm{g}}\right)$ and time scale of microBrownian type molecular motion, respectively, as discussed in the following section.

Dependencies of Molecular Mobility of PMMA Chains (L-Chains) on Matrix Molecular Weight $M_{\mathrm{M}}$ and Tacticity. The narrowing curves of the outermost splitting shifted to higher temperatures with increasing molecular weight $M_{\mathrm{M}}$ of matrix polymer as shown in Figure 2. The remarkable difference of the molecular mobility between the segments located at the end and center of a chain can not be detected. In order to confirm the molecular weight dependence, we observed molecular motion of PMMA chains in binary blends of end-labeled PMMA (L-chains) and atactic PMMA having narrow molecular weight distribution. Figure 3 shows variation of the narrowing curve with molecular weight $M_{\mathrm{M}}$ of the atactic PMMA (M-chains). The curves shift to higher temperatures with increasing the value of $M_{\mathrm{M}}$. For instance, the transition temperature, $T_{50 \mathrm{G}}$ increases with increasing molecular weight of the matrix polymer. The $T_{50 \mathrm{G}}$ are plotted against the reciprocal molecular weight

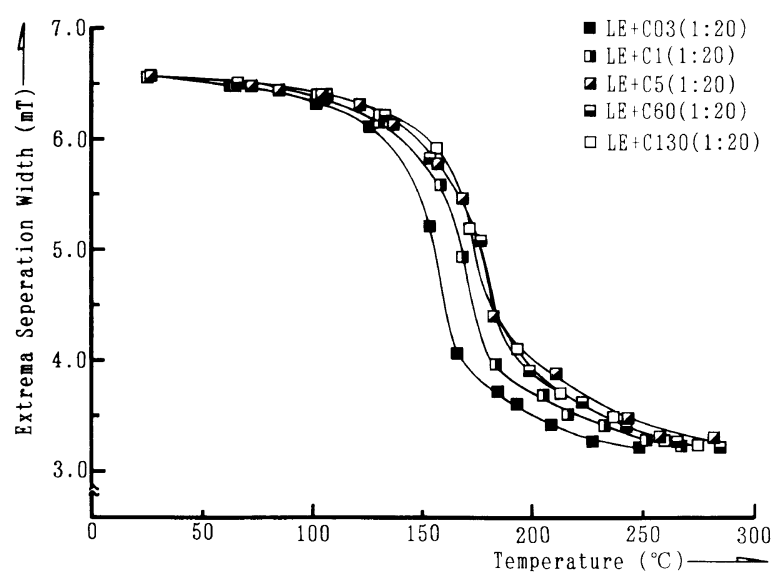

Figure 3. Variation of extrema separation width $\left(2 A_{\mathrm{Z}}\right)$ with temperature: Dependence of the variation on molecular weight $M_{\mathrm{M}}$ of

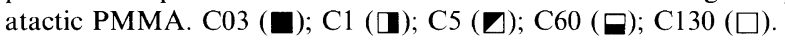

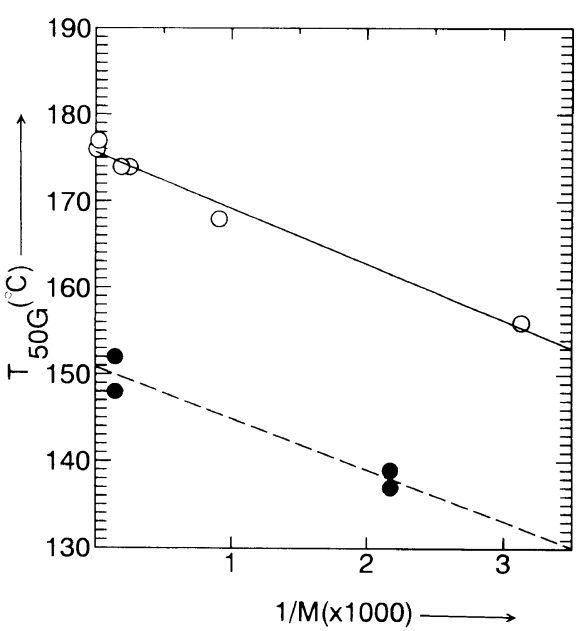

Figure 4. Plots of $T_{50 G}$ of isotactic PMMA (O) and atactic PMMA (O) samples as a function of $M_{\mathrm{M}}{ }^{-1}$.
$\left(M_{\mathrm{M}}{ }^{-1}\right)$ as shown in Figure 4. The linear relationship is similar with that of $T_{\mathrm{g}}$ temperature against the reciprocal molecular weight $\left(M_{n}^{-1}\right)$ given by Fox and Flory. ${ }^{17}$

$$
T_{\mathrm{g}}=T_{\mathrm{g}, \infty}-K / M_{n}
$$

where $T_{\mathrm{g}, \infty}$ is $T_{\mathrm{g}}$ of the polymer with a infinite molecular weight and $K$ is a polymer-specific constant. When the correlation time at $T_{50 \mathrm{G}}$, estimated from ESR data satisfy the WLF equation as discussed in detail in the following section, the difference, $\Delta T$ between $T_{50 \mathrm{G}}$ and $T_{\mathrm{g}}$ should be constant and the eq 1 is converted to

$$
T_{50 \mathrm{G}}=T_{\mathrm{g}, \infty}+\Delta T-k / M_{n}
$$

Ute et al. ${ }^{18}$ recently fractionated highly isotactic and highly syndiotactic PMMA into the individual homologues from the 13 mer to 50 mer by means of supercritical fluid chromatography and fitted plot of $T_{\mathrm{g}}$ vs. $M_{n}{ }^{-1}$ by a linear relationship, eq 1 . They found that the $K$ value, $9.4 \times 10^{4}$ for highly uniform syndiotactic PMMA is smaller than that for atactic PMMA obtained by other workers and suggested that the $T_{\mathrm{g}} \mathrm{s}$ of the non-uniform PMMAs are lower than those uniform PMMAs. The $K$ value, $(6.9-11) \times 10^{4}$ estimated from the linear relationship of $T_{50 \mathrm{G}}$ vs. $M_{\mathrm{M}}{ }^{-1}$ as shown in Figure 4 agrees with that for that obtained by Ute et al. rather than that for non-uniform and atactic PMMAs. These facts suggest that the transition temperature, $T_{50 \mathrm{G}}$ should be related to the temperature, $T_{\mathrm{g}}$ and the characteristic molecular motion of L-chains in matrix polymer with a narrow distribution of molecular weight is detected. It was also found that $T_{50 \mathrm{G}}$ for the matrix polymer of the isotactic PMMA was more than $25^{\circ} \mathrm{C}$ lower and the $K$ value, $5.6 \times 10^{4}$ was smaller than for those for the atactic PMMA. These facts suggests that the configuration effect and structural uniformity are very important factors for the molecular motion.

Dependence of Molecular Mobility of L-Chains on Its Molecular Weight $M_{\mathrm{L}}$. Figure 5 shows variation of the extrema separation width $v s$. temperature curve of the polymer (L-chains) with molecular weight $M_{\mathrm{L}}$. We can estimate molecular mobility of L-chains in binary blends of spin-labeled PMMA with different molecular weight $M_{\mathrm{L}}$ and non-labeled PMMA with molecular weight of $7 \times 10^{4}$. The narrowing curves shift to higher tempera-

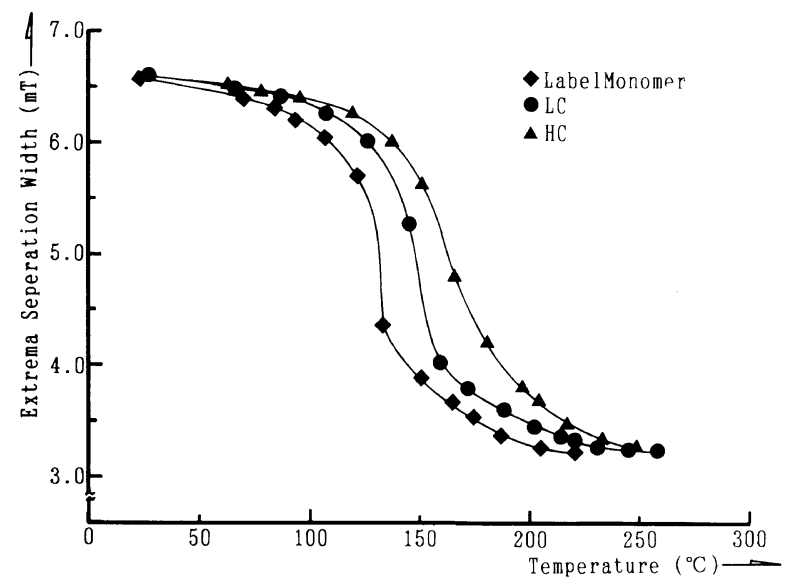

Figure 5. Variation of extrema separation width $\left(2 A_{\mathrm{Z}}\right)$ with temperature: Dependence of the variation on molecular weight $M_{\mathrm{L}}$. Label monomer/non-label (HN) ( $\bullet$ ); center label (LC)/non-label (HN) (O); center label (HC)/non-label (HN) ( $\mathbf{\Delta})$. 
tures with increasing values of $M_{\mathrm{L}}$. This experimental fact suggests that molecular mobility decreases with increasing molecular weight.

Dependence of Molecular Mobility on Distribution of Molecular Weight. Figure 6 shows variations of the extrema separation width $v s$. temperature curve with polydispersity of molecular weight, $M_{w} / M_{n}$ of the matrix polymer. The outermost splitting observed at a temperature is reflected on the average of correlation time and then, for the sake of convenience we use another shape factor, $\Delta T_{\mathrm{w}}$ of the extrema separation width $v s$. temperature curve, i.e., the difference between the temperatures, $T_{60 \mathrm{G}}$ and $T_{40 \mathrm{G}}$ at which the splittings are 60 and 40 Gauss, respectively. The shape factor, $\Delta T_{\mathrm{w}}$ as shown in Figure 6 should be a good measure of the magnitude of a distribution of correlation time according to temperature-time superposition principle. The value of $\Delta T_{\mathrm{W}}$ seems to increase with increasing the polydispersity. Figure 7 shows the dependence of the shape factor on the polydispersity, $M_{w} / M_{n}$, obtained from the Figures 2, 3, and 6. It was found that the distribution of correlation time increased with increasing distribution of molecular weight.

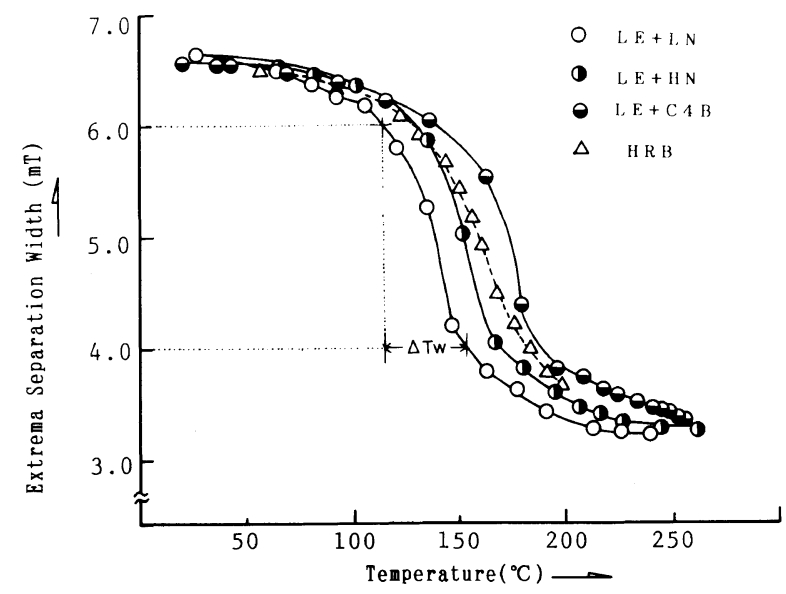

Figure 6. Variation of extrema separation width $\left(2 A_{Z}\right)$ with temperature: Dependence of the variation on polydispersity of molecular weight $M_{w} / M_{n}$.

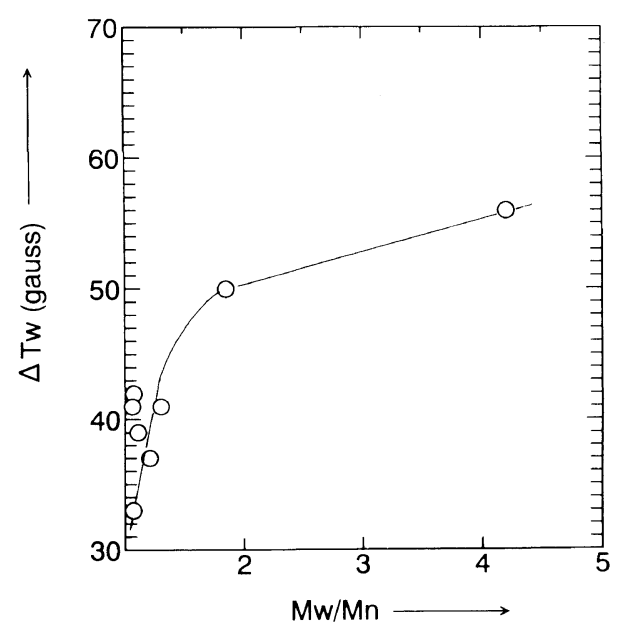

Figure 7. Plots of $\Delta T_{W}$ as a function of polydispersity of molecular weight, $M_{w} / M_{n}$

\section{A Mechanism of Molecular Motion of PMMA Detected by the Spin-Label Method}

Molecular Motion in a Lower Temperature Range. As mentioned in the previous section, the inflection point, $T_{50 \mathrm{G}}$ in the extrema separation width $v s$. temperature curve is a transition temperature detected at an ESR frequency range.

What molecular relaxation mechanism reflects the transition phenomena? In order to answer the question, we should first apply well-known WLF equation, ${ }^{19}$

$$
\log _{10}\left(\tau / \tau_{\mathrm{g}}\right)=-17.44\left(T-T_{\mathrm{g}}\right) /\left(51.6+T-T_{\mathrm{g}}\right)
$$

The representative correlation time, $\tau_{\mathrm{ESR}}$ at the temperature, $T_{50 \mathrm{G}}$ can be calculated from the Freed's equation,

$$
\tau_{\mathrm{ESR}}=1.10 \times 10^{-9}\left(1-2 A_{\mathrm{Z}} / 2 A_{0}\right)^{-1.01}
$$

for "moderate jump diffusion" model, where $2 A_{\mathrm{Z}}$ (50.0 Gauss) and $2 A_{0}$ (68.0 Gauss) are extrema separation width at a certain temperature and that the rigid state, respectively. Then, the $\tau_{\mathrm{ESR}}$ and $\tau_{\mathrm{g}}=100 \mathrm{~s}^{20}$ are substituted into the eq 3 and $\Delta T=T_{50 \mathrm{G}}-T_{\mathrm{g}}$ is calculated to be $74^{\circ} \mathrm{C}$. As well-known, $T_{\mathrm{g}}$ for atactic PMMA is $105^{\circ} \mathrm{C}$. Therefore, the calculated value of $T_{50 \mathrm{G}}, 179^{\circ} \mathrm{C}$ agrees with the experimental value, $177^{\circ} \mathrm{C}$ for the matrix of atactic PMMA with high molecular weight as shown in Figure 4. The estimated time, $\tau_{\mathrm{ESR}}$ is converted to correlation frequency $(f=1 / 2 \pi \tau)$ and plotted in Figure 8 together with correlation frequency for PMMA as measured for dielectric relaxation, dynamic mechanical and nuclear magnetic resonance spectroscopy. ${ }^{21}$

The calculated value, $f_{\mathrm{ESR}}$ agrees with that of microBrownian type molecular motion ( $\alpha$-mechanism). The value of $f_{\mathrm{ESR}}$ is one order of magnitude larger than that of the ester group motion ( $\beta$-mechanism). Velski et al. ${ }^{1}$ and Tsay et al. ${ }^{4}$ also compared ESR data with the results obtained by the other techniques. However, they discussed the relaxation mechanism without consideration of the distribution of correlation times which is a very important factor affecting the relaxation phenomena of polymer substance.

From these discussions, it is concluded that the narrowing behavior of extrema separation width around $T_{50 \mathrm{G}}$ should be reflected on the WLF type, for instance, (a)

O Relaxation Map

- ESR (Averaging of Anisotropic Hyperfine Interaction)

$\Delta$ ESR (Motional Narrowing of Line Width)

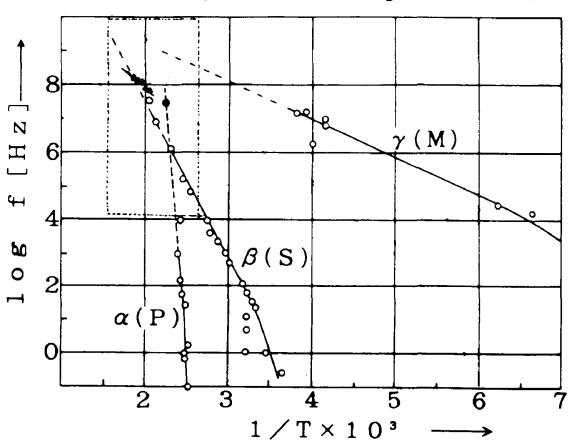

(b)

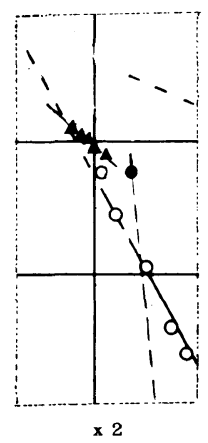

Figure 8. A relaxation map of poly(methyl metacryrate): Plot arranged by Wada $(\bigcirc)$; correlation frequency at $T_{50 \mathrm{G}}$, calculated from ESR data (O); correlation frequency in higher temperature range, calculated by using Kivelson's equation from ESR data $(\boldsymbol{A})$ The dotted part in the figure (a) is magnified by 2 times (b). 
large-scale main chain segmental motion.

Consequently, $T_{\mathrm{g}}$ and the correlation times at temperatures can be estimated from the experimental value of $T_{50 \mathrm{G}}$ by using the eq 3 on the assumption of validity of WLF type behavior for the other PMMA samples. The marks $(O)$ in Figure 9 shows the calculated values of correlation times at $177^{\circ} \mathrm{C} v s$. molecular weight of M-chains of atactic PMMA, whereas the marks (O) in Figure 9 shows the calculated times at $148^{\circ} \mathrm{C}$ vs. molecular weight of L-chains of isotactic PMMA by using the eq 3. (In the present study, we used only the isotactic PMMA with low value of $M_{\mathrm{L}}$ to estimate the effect of molecular weight of L-chains because we could not obtain the spin-labeled and atactic PMMA with high value of $M_{\mathrm{L}}$.)

Current theories ${ }^{22,23}$ predict that the center of mass diffusion of a polymer chain of molecular weight $\mathrm{L}$ in an entangled polymer matrix of molecular weight $M_{\mathrm{M}}$ may occur by three different process: reptation, constraint release, or Stokes-Einstein diffusion. The diffusion coefficient in unentangled matrices $\left(M_{\mathrm{M}}<2 M_{\mathrm{e}}\right)$ is $D_{\mathrm{SE}} \sim M_{\mathrm{L}}{ }^{-0.5} M_{\mathrm{M}}{ }^{-1}$, whereas that in entangled matrices is independent of $M_{\mathrm{M}}$ and proportional to the inverse of $M_{\mathrm{L}}{ }^{2}$.

Here, $M_{\mathrm{e}}$ is the molecular weight between entanglements. Time constant of the diffusion should correspond to correlation time estimated by the spin-label method. At large values of the molecular weight of the matrix, the correlation times, $\tau$ are independent of $M_{\mathrm{M}}$ as shown in Figure 9. This behavior is consistent with diffusion of the L-chain by reptation in a "fixed" environment of constraints. On the other hand, the correlation times seem to be proportional to $M_{\mathrm{M}}$ and $M_{\mathrm{L}}{ }^{0.5}$ in the range of low molecular weight, $M_{\mathrm{L}}$ and $M_{\mathrm{M}}$ as shown in Figure 9. These facts suggest that the molecular motion of PMMA with low molecular weight $\left(M_{\mathrm{L}}, M_{\mathrm{M}}<2 M_{\mathrm{e}} \sim 4 \times\right.$ $10^{4}$ ) observed at $T_{50 \mathrm{G}}$ by the ESR method is attributed to Stokes-Einstein diffusion. Molecular mobility of long chains in entangled matrices and $\mathrm{L}$ dependence should be studied in future.

Molecular Motion in a Higher Temperature Range. The transition phenomena around $T_{50 \mathrm{G}}$ discussed previously

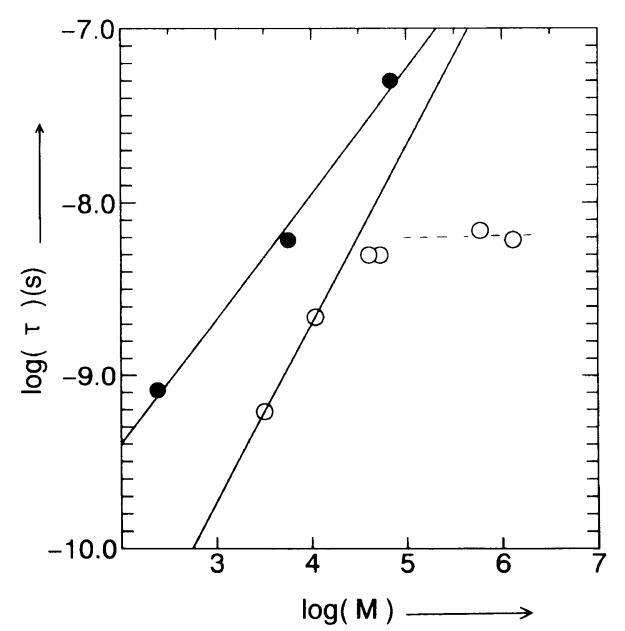

Figure 9. Dependencies of molecular mobility $(\tau)$ of PMMA chains on molecular weight $(M)$. Correlation times, $\tau_{\mathrm{ESR}}$ of L-chains in the matrix of $\mathrm{HN}$ at $148^{\circ} \mathrm{C}$ as a function of its molecular weight $(\bullet$ and correlation times, $\tau_{\mathrm{ESR}}$ of L-chains (LE) at $177^{\circ} \mathrm{C}$ as a function of the molecular weight of the matrix $(O)$. reflect the averaging of the anisotropic interaction between electron and nuclear spins and then, the correlation frequency should be calculated from the anisotropic hyperfine width due to the interaction. We can estimate molecular mobility also from the narrowing of line width of ESR spectrum after the averaging of the anisotropic spin interaction.

What molecular relaxation mechanism reflects the temperature dependence of the line widths of the spinlabeled PMMA?

First, the correlation times are calculated by using Kivelson's equation.

$$
\tau=15 \pi \sqrt{3} W_{0}\left\{\left(h_{0} / h_{1}\right)^{0.5}-\left(h_{0} / h_{-1}\right)^{0.5}\right\} /(8 b \Delta \gamma H)(5)
$$

Here, $h_{1}, h_{0}$ and $h^{-1}$ are respective peak heights of three components. $W_{0}$ is the line width of central component, $H$ is the static magnetic field applied, $b$ and $\Delta \gamma$ in the equation are the quantities for the anisotropic of $g$ - and $A$-tensors. The estimated correlation times for the matrix of atactic PMMA with high molecular weight (the sample of C60) are converted to correlation frequencies and plotted in the so-called relaxation $\operatorname{map}^{20}$ (Figure 8). The calculated values agree with those of the ester group motion ( $\beta$-mechanism) rather than those of $\alpha$-mechanism. This fact suggests that the motional narrowing of line width is reflected on the ester group relaxation.

However, the slope estimated from the spin-label method seems to be smaller than that obtained by the other techniques. This disagreement is responsible for the distribution of the correlation time for the molecular motion. Kivelson's expression is valid for random motion with a single correlation time. Figure 10 shows examples of plots of logarithmic correlation times against the reciprocal temperatures. The activation energies estimated from the slope decrease with increasing polydispersity of molecular weight, $M_{w} / M_{n}$ as shown in Table II. The potential barrier for molecular rotation of the ester side group should be strongly affected by the surrounding environments, i.e., inter molecular interaction, segmental density, steric hindrance, and etc. However, it can be considered that the polydispersity of molecular weight of PMMA with the same value of $M_{n}$ does not affect the surrounding environments. Therefore,

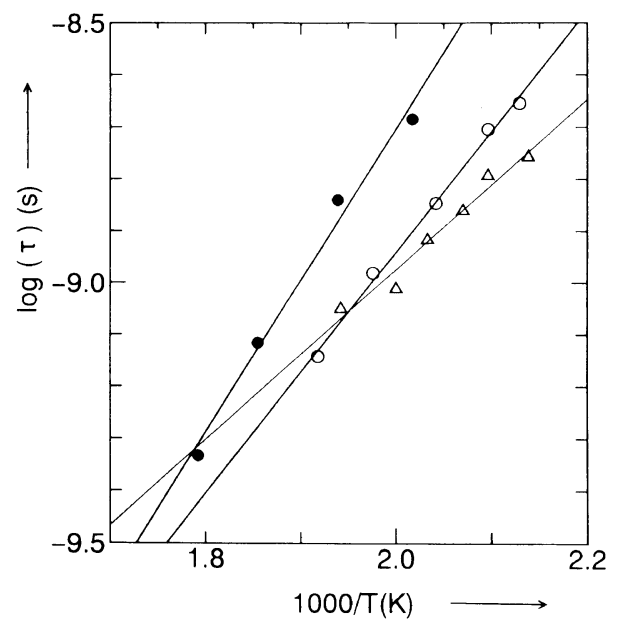

Figure 10. Examples of temperature dependencies of correlation time of spin-labeled PMMA: LE/C60 (๑); LE/HN $(O)$; HRB $(\triangle)$. 
Table II. Activation energies estimated from temperature dependencies of correlation time (Figure 10)

\begin{tabular}{lcc}
\hline Sample & $M_{w} / M_{n}$ & $E_{\mathrm{a}} / \mathrm{kcal} \mathrm{mol}^{-1}$ \\
\hline LE/C60 & 1.04 & 13.4 \\
LE/HN & 1.28 & 9.2 \\
HRB & 4.20 & 7.3 \\
\hline
\end{tabular}

$M_{w} / M_{n}$ dependence of the activation energy as shown in Figure 10 is caused mainly by the distribution of molecular weight which bring about the structural distribution, and the distribution of correlation times.

In general, the activation energy estimated by the magnetic resonance method should be both functions of the magnitude of the distribution and the potential barrier of molecular motion. The anisotropic rotation of the side group from the spectrum simulation of the spin-labeled PMMA was studied by Shiotani et al. ${ }^{6}$ For the sake of brevity, the anisotropic rotational correlation times, $\tau_{\|}$and $\tau_{\perp}$ and the degree of anisotropic rotational reorientation, $N=\tau_{\|} / \tau_{\perp}$ are calculated by using Freed's equation. ${ }^{24}$ Here, $\tau_{\|}$and $\tau_{\perp}$ are rotational correlation times about major and minor axes of the axially symmetric rotor. The average correlation times, $\sqrt{\tau_{\|} \tau_{\perp}}$ have similar value to that based on the isotropic rotational model (eq 5). It is well-known that the major axis is parallel to the direction of the $\mathrm{N}-\mathrm{O}$ axis (approximately perpendicular to the main chain axis). The values of $N$, which is calculated to be $2-3$ in the higher temperature range, increase with increasing temperature. For instance, the rotational motion around the major axis can be a slow rotation of the ester side group in comparison with the rotation around other axes. The motional narrowing of ESR spectrum can be observed when the time constant corresponding to the ESR line width is comparable to that of the slow motion. These facts support the molecular motion of the spin-labeled
PMMA in the higher temperature range is attributed to the rotation of the ester side group.

\section{REFERENCES}

1. S. Shimada, Prog. Polym .Sci., 17, 1045 (1992).

2. Z. Veksli and W. G. Miller, Macromolecules, 10, 686 (1977).

3. A. T. Bullock, G. C. Cameron, and V. Krajewski, J. Phys. Chem., 80, 1972, (1976)

4. M. Shiotani and J. Sohma, Polym J., 9, 283 (1977).

5. F. Tsay and A. Gupta, J. Polym. Sci., Polym. Phys. Ed., 25, 855 (1987).

6. S. A. Goldman, G. V. Bruno, and J. H. Freed, J. Phys. Chem., 76, 1858 (1972).

7. D. Kivelson, J. Chem. Phys., 33, 1094 (1960).

8. M. Shiotani and J. Sohma, Macromolecules, 16, 1495 (1983).

9. K. Murakami and J. Sohma, Polym J., 11, 545 (1979).

10. F. Tsay, S. D. Hong, J. Moacanin, and A. Gupta, J. Polym. Sci., Polym. Phys. Ed., 20, 763 (1982).

11. G. G. Cameron, I. S. Miles, and A. T. Bullock, Br. Polym. J., 19, 129 (1987).

12. N. Bloembergen, E. M. Purcell, and P. V. Pound, Phys. Rev., 73, 679 (1948).

13. S. Shimada, T. Tanigawa, and H. Kashiwabara, Polymer, 21, 1116 (1980).

14. S. Shimada, T. Tanigawa, and H. Kashiwabara, Appl. Spect., 34, 575 (1980).

15. K. Hatada, K. Ute, K. Tanaka, T. Kitayama, and Y. Okamoto, Polym. J., 17 (1985).

16. T. Kurosaki, K. W. Lee, and M. Okawara, J. Polym. Sci., Polym. Chem. Ed., 10, 3295 (1972).

17. T. Fox and P. J. Flory, J. Appl. Phys., 21, 581 (1950).

18. K. Ute, N. Miyatake, and K. Hatada, Polymer, 36, 1415 (1995).

19. J. D. Ferry, "Viscoelastic Properties of Polymers," John Wiley \& Sons, New York, N.Y., 1960.

20. R. A. Komoroski, "High Resolution NMR Spectroscopy of Synthetic Polymer in Bulk," VCH Publishers, Florida, 1986.

21. Y. Wada, J. Phys. Soc. Jpn., 16, 1226 (1961).

22. P. F. Green and E. J. Kramer, Macromolecules, 19, 1108 (1986).

23. M. Doi and S. F. Edward, "The Theory of Polymer Dynamics," Oxford University Press, Oxford, 1986.

24. S. A. Goldman, G. V. Bruno, C. F. Polnaszek, and J. H. Freed, J. Chem. Phys., 56, 716 (1972). 